\title{
La memoria del lugar: lo extraño y la forclusión en Gregor Schneider
}

\begin{abstract}
Resumen
Nuestros objetivos en este artículo son, primeramente, abordar la investigación de la obra de Gregor Schneider como caso de estudio de arte de instalación, dentro de las manifestaciones del arte contemporáneo que utilizan recursos espacio-temporales. Seguidamente, vemos que la obra de Gregor Schneider es paradigmática en este sentido, ya que posibilita la transformación de las estructuras espaciales y arquitectónicas, en un ejemplo de cómo los artistas contemporáneos proponen nuevas vías para la definición de la instalación escultórica. Y finalmente, exploramos su obra a partir de los estudios visuales para intentar proponer nuevas formas de entendimiento de la obra, en relación al arte de instalación performativa y espacial. Metodológicamente, estructuramos la investigación en dos partes. En la primera parte, nos servimos del concepto de Freud de lo "uncanny", para entender la duplicación de los espacios en la obra de Gregor Schneider. Intentamos responder a la cuestión principal de su obra, el porqué de la duplicación de espacios. Entendiendo esta duplicación a través del término lacaniano de la "forclusión", nos centramos en sus obras Haus u $r$ y Die Familie Schneider. En una segunda parte, analizamos y cuestionamos el papel que desempeña la idea de la memoria y la idea del trauma en relación al contexto en el que está situada su obra. Utilizamos los conceptos de la memoria, el tiempo, el trauma y los lugares desarrollados por Dylan Trigg como elementos que dan sentido a su obra. Por último, concluimos con la reconstrucción de la memoria desarrollada en las obras de Gregor Schneider, donde la idea de trauma implica una teatralización y una activación perceptiva del espectador.
\end{abstract}

\author{
Jesús Segura Cabañero \\ Doctor en Bellas Artes \\ Profesor de la Universidad de Murcia \\ Murcia, España. \\ Correo electrónico: jesusegu@um.es \\ () orcid.org/0000-0003-3708-4140 \\ Google Scholar \\ Toni Simó Mulet \\ Doctor de Bellas Artes \\ Profesor de Universidad de Murcia \\ Murcia, España. \\ Correo electrónico: simo@um.es \\ - orcid.org/0000-0002-0917-9938 \\ Google Scholar
}

Recibido: abril 12 de 2019 Aprobado: marzo 26 de 2021

Palabras clave:

forclusión, Gregor Schneider, lugar, memoria, tiempo. 


\section{The Memory of Place: the Strange and Foreclosure in Gregor Schneider}

\begin{abstract}
The objectives in this article are: firstly, to approach the research of Gregor Schneider's work as a case study of installation art within the manifestations of contemporary art that use spacetime resources; secondly, Gregor Schneider's work is seen as paradigmatic in this sense, since it enables the transformation of the spatial and architectural structures, in an example of how contemporary artists propose new ways to define the sculptural installation; and, thirdly, Schneider's work is explored from the point of view of visual studies to try to propose new ways of understanding his work in relation to the art of performative and spatial installation. Methodologically, the research is structured in two parts. In the first part, Freud's concept of the "uncanny" is used to understand the duplication of spaces in the work of Gregor Schneider. An answer to the main question of his work, the reason for the duplication of spaces,I s intended. Understanding this duplication through the Lacanian term "foreclosure", the focus is mainly on his works Haus u $r$ and Die Familie Schneider. In a second part, the role played by the idea of memory and the idea of trauma in relation to the context in which his work is situated are analyzed and questioned. The concepts of memory, time, trauma and places developed by Dylan Trigg are used as elements that give meaning to his work. Finally, the work is concluded with the reconstruction of memory developed in the works of Gregor Schneider, where the idea of trauma implies a theatricalization and perceptual activation of the viewer.
\end{abstract}

Key words:

foreclosure, Gregor Schneider, place, memory, time. 


\section{Introducción}

"Lo desacogedor: Escenas fantasmas en la sociedad global" fue el título de la "Bienal Internacional de Arte Contemporáneo de Sevilla" de 2006. La temática general de la bienal fue sintomática de la era global en la que nos encontramos, y reproducía de alguna manera una constante desde hace décadas sobre las preocupaciones de los artistas contemporáneos.

El tema de las ansiedades — sean estas políticas, sociales, psicológicas o culturales - era tratado bajo temas que comprendían la sociedad de control y su administración global o la lucha contra el terrorismo global. De lo que se derivaba un segundo tema que se presentaba como causalidad del primero: el miedo y el poder ligado a la sociedad de control y las restricciones de las libertades. Haciendo especial hincapié en lo "desacogedor" en el mundo globalizado, debido a la transformación de los aparatos del poder y del control global (Enwezor, 2006, pp. 13-14). Es decir, los nuevos escenarios del capitalismo global se reformulan y generan nuevos espacios-tiempos sociales, económicos y políticos desfigurando la realidad, mediante una estrategia de "desfamiliarización".

Las metamorfosis de la "desfamiliarización" generan escenas fantasmales, nuevos espacios-tiempos reconfigurados, alterados, y extraños a la percepción de nuestra realidad cotidiana. No es casualidad que la escena del arte contemporáneo trate estos temas en la contextualización de los proyectos artísticos. Las escenas fantasmales de la globalización son un producto más en nuestra "era de la ansiedad" o en nuestra "cultura del miedo".

En este sentido, nuestra investigación toma como punto de partida esta derivación temporal que habilita un contexto espaciotemporal alterado, trastocado y desquiciado (Bal, 1999) que genera una percepción desafectada, extraña y siniestra, para configurar un estudio sobre los nuevos modelos 
espacio-temporales que el arte contemporáneo presenta como experimentos utópicos. El trabajo artístico de Gregor Schneider es paradigmático en este sentido y se basa en la manipulación del espacio arquitectónico y su percepción sensorial. Su producción artística se podría categorizar como instalaciones espaciales, performance y acciones en directo.

El artista fundamenta su creación artística a partir de la exploración de un lugar concreto, trabajando sobre lo perceptivo y lo sensitivo, sobre lo visible y lo invisible; donde la percepción y la duplicidad de los espacios se contraponen al paso del tiempo y al desvanecimiento de la experiencia vivida, junto al devenir y decadencia del entorno que plantea las preguntas sobre el espacio, el trauma y la memoria. Así, examinamos en este texto el extrañamiento, lo uncanny, la duplicación el espacio, el trauma y la memoria que habita en lo cotidiano, como figuraciones contemporáneas de lo siniestro y lo extraño. Este proceso lo encontramos en el trabajo del artista Gregor Schneider.

\section{Objetivos}

- Definir un caso de estudio de producción artística como una oportunidad para elaborar un discurso sobre las instalaciones artísticas contemporáneas que utilizan los recursos espacio-temporales.

- Analizar la obra de Gregor Schneider a partir de un estudio y aproximación interdisciplinar, para entender cómo las estructuras espaciales y arquitectónicas de su trabajo se pueden convertir en una propuesta de instalación escultórica.

- Examinar la obra de Gregor Schneider a través de disciplinas dentro de los estudios visuales como, por ejemplo, el psicoanálisis, los estudios performativos y espaciales, los estudios filosóficos y estéticos de la 
memoria y los lugares para sugerir nuevas interpretaciones sobre la significación de la obra de Gregor Schneider.

\section{Metodología}

Para abordar la investigación sobrelas manifestaciones artísticas contemporáneas del arte de instalación nos hemos basado en un caso de estudio concreto, y hemos analizado la obra del artista Gregor Schneider. Su obra nos permite indagar sobre una serie de conceptos que están presentes a lo largo de su trayectoria como artista de referencia internacional. Su insistencia en las estrategias de intervención espacial, manipulación temporal y performatividad nos conduce a proponer una serie de autores con los que debatir su obra, utilizando la metodología de la cultura visual.

De esta manera, dividimos en dos partes el análisis de la obra de Schneider. En la primera parte utilizamos el concepto de uncanny de Freud (1919) para profundizar en su trabajo. Lo uncanny, que puede ser traducido como extraño, siniestro, asombroso, fantasmagórico, etc., participa de la obra de Schneider en su poética artística, que es la duplicación y la repetición de espacios. Este recurso artístico de instalación espacial artística de la duplicación representa, según nuestra interpretación crítica y visual, la figura de la "forclusión", término utilizado por Lacan y ligado al concepto elaborado por Freud de lo "uncanny".

Así, también, nos servimos de los conceptos descritos por Gaston Bachelard (2012) sobre el espacio de la casa como unidad habitable y representable. Exploramos también el concepto de la performatividad de los espacios duplicados de Schneider con las aportaciones de Philip Auslander (2003). Según Auslander, Schneider trabaja sobre la idea temporal del espacio basado en la memoria y en las huellas que deja esta, por tanto, incorpora una actuación teatral en el pasado, así como en el presente de la obra duplicada. 
En una segunda parte, analizamos la estrategia del doble espacial en Schneider y su relación con la idea del trauma y la memoria. La memoria es el concepto clave para entender la duplicación del espacio en las obras de Schneider. Recurrimos al contexto en el que se ubica su obra, en la zona de la ciudad de Rheydt, donde la mayoría de las casas fueron arrasadas en la Segunda Guerra Mundial. Y apuntamos, siguiendo los conceptos de Mark Crinson (2005) de la "memoria urbana", que su obra reactiva el concepto de memoria antropomórfica del lugar y posibilita la recreación de la casa familiar como lugar performativo.

Siguiendo a Halbwachs (2011), proponemos que el trabajo de reconstrucción e interpretación de los espacios de la obra Haus u $r$ de Schneider en realidad desvela una crítica simbólica a la reconstrucción de la Alemania de posguerra. Con el uso conceptual de la temporalidad espacial, la obra de Schneider se nos presenta como el inconsciente simbólico del trauma de los efectos de la guerra, y su reconstrucción posterior de la ciudad y sus edificios.

Debatimos, siguiendo las teorías de Dylan Trigg (2009) sobre la memoria, el espacio y el trauma, y proponemos que la experiencia traumática de posguerra está en el subconsciente del trabajo de Schneider, cuando aborda las reconstrucciones de espacios, interpretando los espacios familiares de la casa como huellas y ruinas de ese mismo trauma. Esta idea del trauma elaborada por Trigg cuestiona la unidad temporal y espacial de la memoria del lugar. De la misma manera, Schneider interrumpe una narrativa lógica y temporal de sus espacios familiares al multiplicar de manera incesante y no lógica, es decir no cronológica. A partir de la idea del trauma de Dylan Trigg recomponemos la importancia de la idea de la experimentación del espectador en la obra de Schneider; sin esta experiencia del espectador su obra no queda completa. 


\section{The uncanny (lo extraño): el espacio forcluído}

En el trabajo de Schneider podemos ver la presencia de lo siniestro como una emoción estética basada en la repetición involuntaria, o como una emoción real que presenta una intrincada relación con la figura de la repetición y del doble. En la teoría psicoanalítica se afirma que los impulsos emocionales se convierten en miedo a lo desconocido al ser reprimidos. A partir de aquí, se puede deducir que entre las cosas que son aterradoras puede haber algunas en las que se puede demostrar que el elemento aterrador es algo que ha sido reprimido y regresa como repetición, como doble. Y, es así como Freud (1919) define el tipo de emoción de "lo siniestro, como una forma particular de miedo que se deriva de la devolución de algo reprimido" (p. 240).

Esta emoción de lo extraño, lo siniestro, en realidad no es ninguna novedad, sino algo que era muy familiar para la psique y se había alejado de ella solo a través de la represión. Así pues, la obra de Gregor Schneider está impregnada por lo que hemos denominado "Ios espacios forcluídos". Entendiendo estos, en el estricto sentido lacaniano (Lacan, 1988), como "espacios escotomizados", o lo que es lo mismo: "espacios de represión".

Es a partir del proyecto Haus u r, en Rheydt, Alemania (Figura 1), que empezó en 1985, donde se producen esos debates con el espacio. Se trata de una acción performativa sobre el espacio donde se articula una reconstrucción, deconstrucción, manipulación y duplicación de las habitaciones de la casa. De hecho, Schneider afirma:

Mi método de trabajo es siempre el de duplicar. Un doble justo en frente, justo por debajo o justo en el interior de lo que ya existe, o un doble plausible colocado en otro sitio. Así que no hay invención. Lo poco que invento es apenas perceptible y discreto. La duplicación es un gesto que limita lo que ya existe en el presente, no en la forma de una declaración o prueba, sino como prueba en un tribunal de justicia. (Williams, 2010, p. 1) 


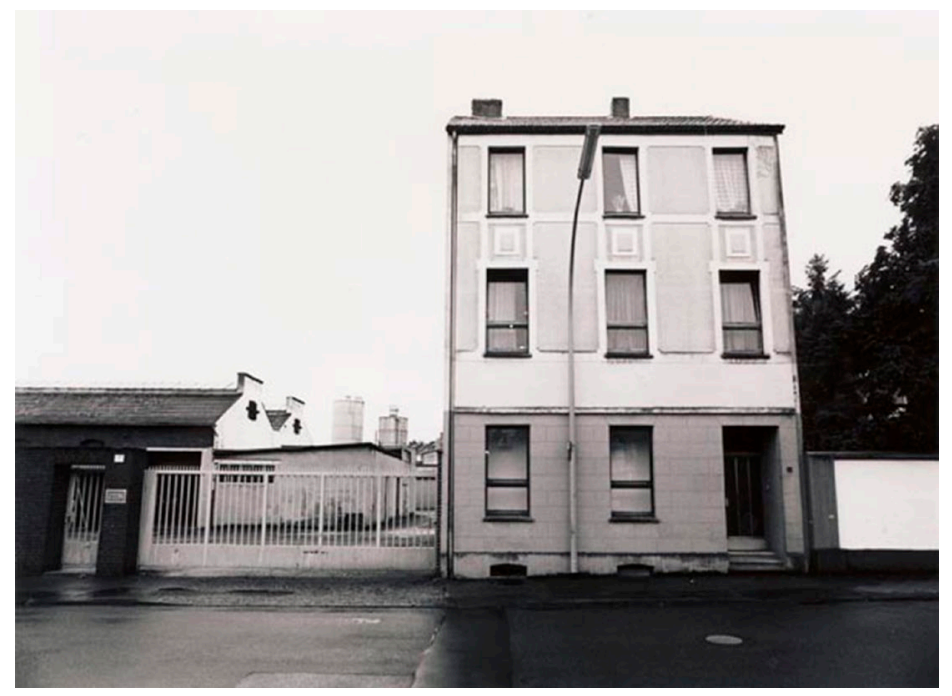

Figura 1. Gregor Schneider, Haus u r, Rheydt, Alemania, 1985 - actualidad. (C) Gregor Schneider/VG Bild-Kunst Bonn.

La duplicación en la obra de Schneider (Figura 2) aparece como un proceso, una herramienta sencilla de elaboración espacial, una técnica artística que con el tiempo a base de repetir la misma acción se convierte en algo que va descubriendo la naturaleza del espacio. Así, la duplicación para Schneider es un acto de repetición para acceder a un conocimiento diferente del espacio:

Del mismo modo que se puede contemplar una pared durante horas. Cuando se hace una vez, dos veces, un mes entero o más tiempo aún, Ilega un momento en que se puede hablar a todo el mundo de esa pared. (Schneider et al., 2011, p. 130) 


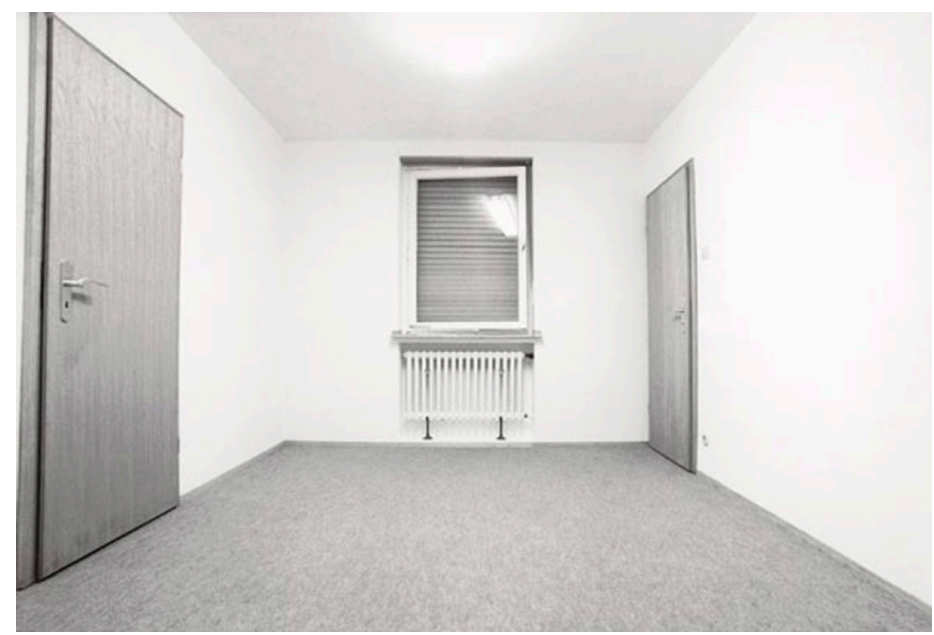

Figura 2. Gregor Schneider, u r 1. Habitación dentro de una habitación, paredes blancas y techo, separado, 30-50 cm de distancia desde la habitación exterior $(298 \times 396 \times 249 \mathrm{~m})$, Haus u r, Rheydt, Alemania, 1985 - actualidad. (C Gregor Schneider/VG Bild-Kunst.

Fuente: https://tinyurl.com/wmhr3z9k

Pero sin duda, la repetición y duplicación de Schneider es la clave de su obra, y esta sigue las teorías de lo uncanny de Freud como una forma para la manifestación de los temores y las experiencias reprimidas. La preocupación de Schneider por las atmósferas cargadas psíquicamente está presente a lo largo de toda su obra. Su predilección por lo siniestro, por los lugares marcados por el horror y lo espantoso se puede ver en las habitaciones vacías y neutras, a través de rastros antropomórficos que deja en las habitaciones duplicadas; como los objetos cotidianos y los muñecos con bolsas de plástico que parodian la muerte. 
En este sentido, el espectador percibe el vacío y la neutralidad de sus espacios con ansiedad e inquietud, porque cualquier cosa puede suceder. La ansiedad y la muerte son conceptos tratados por Freud (1919) en el uncanny, donde la repetición y el doble actúan como instinto de supervivencia (pp. 234-235). El hecho de duplicar, de traer a la vida algo inanimado por el acto de la duplicación, es para Freud la autoafirmación del ego desde la niñez, para, de este modo, superar el miedo a la muerte y al vacío.

La duplicación sirve para perpetuar el sentimiento de vida de objetos, situaciones y personas. El doble es una expresión de la preservación y actúa contra la extinción, "está conectado con reflejos en espejos, con sombras, con espíritus guardianes, con la creencia en el alma y con el miedo a la muerte" (Freud, 1919, p. 235). Ya que el doble es la seguridad para la continuación del ego, la duplicación se convierte en un acto psíquico de autoafirmación y prolongación de la vida, aunque sea mediante la reanimación y la repetición como un acto compulsivo y obsesivo, como lo vemos representado en la obra de Schneider. Schneider trabaja el interior y exterior de los espacios de la casa como un objeto vivo, como una casa viviente. Los recuerdos de su infancia quedan atrapados dentro de ella y establecen una lucha permanente entre los cambios y movimientos de las estructuras de la casa.

Quizás, el ejemplo más claro de reanimación y duplicación de la casa sea la manera como expande el trabajo Haus u r en las galerías de arte, transportando las habitaciones y reanimando los espacios en la sala de exposiciones. El organismo viviente de la casa se resquebraja y se extirpan los órganos para ser mostrados en otros espacios expositivos. El espectador, ante estas reanimaciones y duplicaciones, puede tener un sentimiento uncanny sobre la propia naturaleza de lo familiar, preguntándose si son parte de la casa familiar original, o si son espacios vividos, o contienen algún elemento antropomórfico que puedan identificar como espacios de vida. 
Esta incertidumbre sobre lo animado y la duplicación es una de las características de lo uncanny a la que alude Freud cuando comenta, siguiendo a Jentsch, que se producen unas condiciones favorables para emerger sentimientos de lo uncanny: "Cuando hay incertidumbre intelectual sobre si un objeto está vivo o no, y cuando un objeto inanimado se vuelve demasiado parecido a un objeto animado" (Freud, 1919, p. 233).

Para los espectadores que entran en la casa, la estructura original no es visible, pero son conscientes de que es la casa familiar del artista y que los recuerdos y las vivencias quedan atrapados en su interior. El espectador se enfrenta de alguna manera con "lo desconocido" del espacio familiar anterior, y en algunos casos Schneider deja deliberadamente fotografías y objetos de su vida familiar en la casa, en los espacios que quedan detrás de las paredes reconstruidas de las habitaciones. Este atrapamiento interior del pasado forma parte de la estrategia de reanimación y duplicación de los espacios que Schneider ejecuta con precisión. La carga psicológica de los espacios de Schneider se debe, en parte, a la sensación de que los espacios inaccesibles, escondidos dentro de las habitaciones duplicadas, desorientan al espectador. Y este es también un efecto, según Freud, de lo uncanny (Freud, 1919, p. 221).

El acto de duplicar de Schneider se puede interpretar como un acto instintivo de supervivencia, pero también como un acto estético de creación. Y en última instancia, se puede interpretar como un proceso escultórico de representación tridimensional entre el modelo y el original o la copia o reproducción (Moriente, 2007, p. 192). En el texto de Freud, el concepto de uncanny también denota una emoción explícita real que existe, pero que se constituye estéticamente. Aquí, el concepto estético y emocional de lo uncanny se mezcla con la manifestación del miedo y sus represiones. Podríamos plantearnos cómo funcionan los espacios repetidos de Schneider en el espectador, y cuál es el motivo que hay detrás de esa obsesión en Schneider por la duplicación. 
Finalmente, la duplicación de espacios transmite al espectador la distinción entre lo "real" y lo "fantástico", de lo siniestro, interrumpiendo la percepción familiar de un espacio doméstico, como algo completamente extraño y alienado. La tentativa de Schneider de deshacer y reconstruir espacios, es decir, la función del doble en Schneider, es el intento de resucitar y rescatar para la vida, para lo animado, algo que reposa en la memoria del artista en el lugar, como algo familiar. Schneider, a través de la duplicación, reproduce un mecanismo de defensa para combatir el miedo a la muerte y a la desaparición. En este sentido, la duplicación como espacio forcluído, reprimido, es la herramienta que utiliza Schneider para centrarse en la posibilidad de la memoria de un lugar, en este caso la casa familiar en Reydht.

Haus u r es la casa donde el artista pasó la infancia, ubicada en la ciudad donde nació, en Rheydt, Alemania. Schneider transforma continuamente las habitaciones de la casa en espacios dentro de espacios, que son réplicas de las anteriores. Creando así un laberinto de espacios misteriosos que son alterados por los rastros de presencia que deja de las anteriores habitaciones. El espectador transita por el interior de la casa encontrándose una habitación tras otra sin ninguna conexión lógica, intentando descifrar una espacialidad amorfa y orgánica. El visitante se enfrenta a una serie de espacios que desafían su compresión del lugar.

Philip Auslander (2003) nos habla de la performatividad de los espacios de Schneider intentando comprender la función que estos desarrollan en su obra. Así, destaca que hay dos funciones teatrales en el proyecto Totes Haus u r, 2001 (Figura 3) que hizo para la Bienal de Venecia. Una primera función escenográfica y otra segunda como actor, cuyo propósito es habilitar un entorno para crear una expresividad sobre la memoria y hacer emerger las huellas del mismo lugar. En definitiva, suspender la presencia inquietante de algo que puede ser percibido y sentido pero que es complemente invisible, o por decirlo de otra manera, está "performativizado". 
Como ha señalado Philip Auslander (2003), Schneider trabaja sobre los fenómenos ocurridos en el pasado, mediante la memoria y la emoción como señal cargada psicológicamente:

Este espacio fomenta a su audiencia a renunciar a su posición puramente pasiva y los obliga a convertirse en actores también [...] La casa en su conjunto es una escenografía; cargada de memorias. Todos estos elementos se encuentran en el límite o más allá de la percepción de los espectadores, sin embargo, influyen en la experiencia afectiva del espacio por parte de los espectadores. (p. 87)

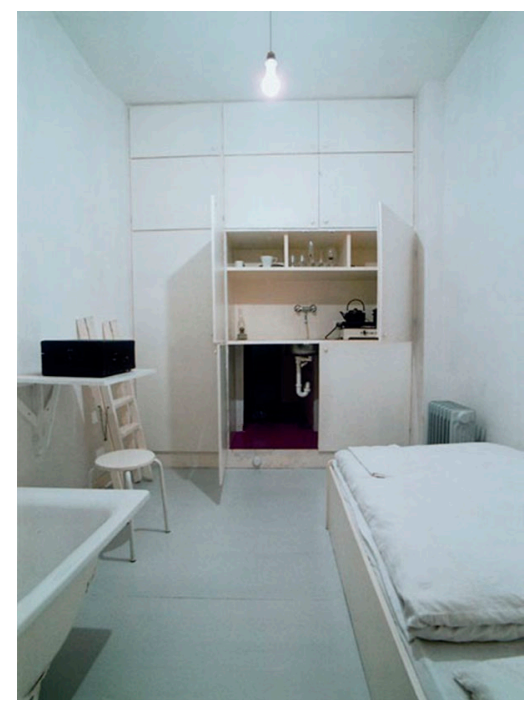

Figura 3. Gregor Schneider, Totes Haus u r, Rheydt, Alemania, 1985 - Venecia, 2001 Habitaciones construidas $(8,5 \times 18,5 \times 22$ m), Pabellón de Alemania, 49 Exposición Internacional de Arte, la Biennale di Venezia, Venecia, Italia. (C) Gregor Schneider/VG Bild-Kunst. Fuente: https://tinyurl.com/4s58en2j 
Gaston Bachelard (2012) explora la poética del espacio y las relaciones que existen entre lugar, espacio y memoria, y más concretamente el espacio habitado de la casa. La casa se puede considerar como un espacio principal en la experiencia personal, "es nuestro primer universo", y constituye nuestras primeras experiencias psíquicas, perceptivas y espaciales, porque es "realmente un cosmos". Además, los espacios interiores de la casa son archivos de historias íntimas. No solo nuestra memoria, "sino también nuestros olvidos, están alojados en los espacios de la casa" (p. 23).

En el Proyecto Haus u r, Schneider trae al primer plano los temas de la historia, la memoria colectiva y la memoria personal. Pero también las cosas que han sido olvidadas, reprimidas y forcluídas en el espacio simbólico del hogar. Meticulosamente, apunta todos los cambios de las habitaciones y los enumera para tener un seguimiento. Sin embargo, él ha manifestado que no sabe desentrañar la lógica de los espacios y no recuerda qué había antes o al otro lado de la pared. No consigue recordar el paso del tiempo en el hogar. En este sentido, trata la casa como una "geometría distópica" y atemporal (Schneider et al., 2011, p. 76).

Por tanto, lo temporal juega un rol importante en su trabajo, ya que principalmente desarrolla su proyecto a partir de la acción libre. Como él mismo comenta: "Estoy interesado en la acción libre. Mi trabajo es una adición - algo extra o superfluo en el sentido de que no dice, o expresa, o se refieren a nada nuevo" (Williams, 2010, p. 1). La sucesión de habitaciones dentro de habitaciones hace que "uno se encuentre dentro de un imperceptible marco de tiempo completamente alienado" (p. 2). Su acción libre comienza en la pared. La pared como aquello que contiene la performance espacio-temporal, en la que el propio artista recuerda el espacio anterior con la acción de tapiar, esconder, hacer un simulacro del mismo espacio y almacenar los recuerdos de la casa. 
En la escenificación de sus espacios, el cuerpo toma relevancia como perceptor y habitante de la transformación escenográfica que conforma el acontecimiento. Schneider trabaja en el manejo de la temporalidad subyacente en la casa, y esto le permite destruir y reconstruir permanentemente las habitaciones. La contradicción de su acción performática de negar y posibilitar nuevos espacios, de hacer efímera cualquier posibilidad perceptiva estable y transmitir una carga psíquica continuada, solo se puede entender si la intencionalidad del artista se sitúa entre estas dos posibilidades, que permiten al espectador una experiencia del rastro y ruina que dejan sus espacios.

Esta definición de la obra de Schneider se sitúa en el "entre", en la hibridación espacio-temporal del espacio doblado con el original, y representa una reconsideración del mismo lugar y el lapso que les separa. Como apunta Dionne (2003): "Es como si, en cada uno de estos espacios algo había acabado de suceder, o estaba a punto de ocurrir" (p. 30).

Una de las cosas que intenta Schneider es elevar la percepción del espectador, al explorar el vacío que deja al duplicar los espacios. La duplicidad se extiende a la ambigüedad de la percepción del espectador y, al mismo tiempo, a la situación real de sus espacios, vistos como obra de arte, o como espacios funcionales de la vida cotidiana. La dualidad se multiplica en la percepción de sus obras. Como él mismo apunta, hablando sobre la intencionalidad de su trabajo:

Bueno, la cuestión aquí es, visible/invisible, reconocible/irreconocible. Una habitación construida dentro de una habitación es visible, pero no reconocible. Eso significa, que lo ves como una habitación normal, pero no la reconoces como una obra de arte. Por lo tanto existe una brecha. Mis obras son muy complicadas. Las situaciones difieren para cada visitante, y cada visitante tiene su propia idea acerca de las habitaciones. Solo puedo decir que los visitantes serán guiados a pie por el interior de mis obras y se preguntarán, ¿Es esta alguna clase de obra de arte? ¡Al final, no hay ninguna pregunta más sobre el arte! La diferencia entre la vida y el arte no existe más. (Kobayashi e Inoue, 2014, párr. 23) 
Una de las piezas, por ejemplo de Haus u r, es la habitación Kaffeezimmer, 1993 (Figura 4), donde la habitación duplicada no es visible para los espectadores, y todos los elementos del espacio son una copia exacta de la habitación que está detrás de las paredes. La escenografía se completa con una ventana falsa, una luz artificial y una mesa, donde el artista invita al visitante a tomar café y a experimentar el espacio. La habitación, muy lentamente, va girando sobre su eje con un mecanismo situado en la base, de manera que el movimiento es casi imperceptible. La representación radica en esta duplicidad de:

La pared delante de la pared, el techo debajo del techo, piso en piso, habitación en habitación. Es una labor de representación que utiliza los mismos materiales o similares para replicar en el mismo lugar algo que ya existe allí, bajo una o más capas. (Loock, 2002, p. 143)

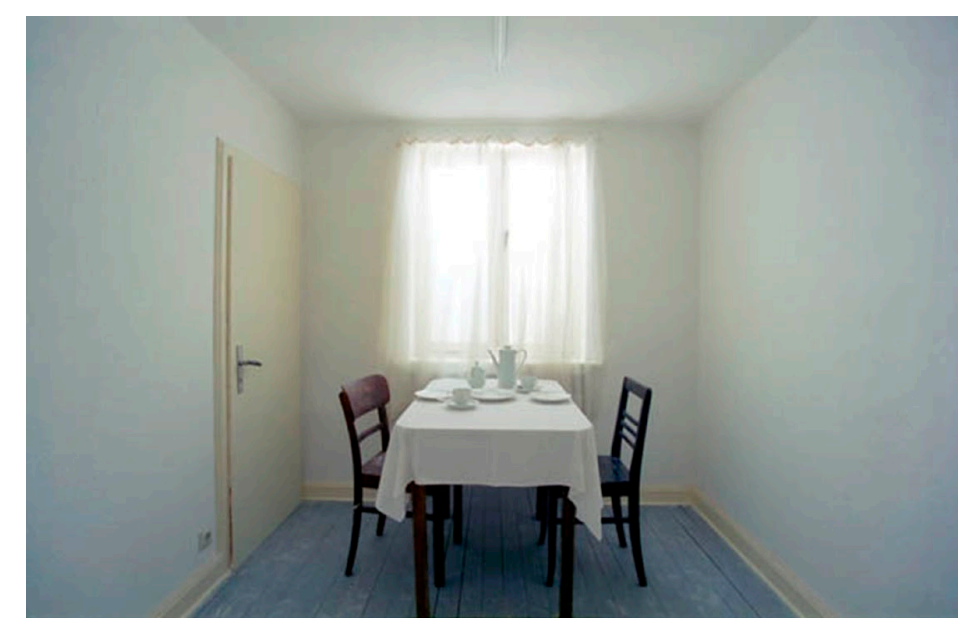

Figura 4. Gregor Schneider, U r 10 Kaffeezimmer, 1993, Haus u r, Rheydt, Alemania, 1985 - actualidad. (C) Gregor Schneider/VG Bild-Kunst. Fuente: https://tinyurl.com/tzn275sj 
Gregor Schneider ha tratado muchas veces en su obra este efecto denominado "doppelgänger": la duplicación de lo real existente, como por ejemplo en el proyecto Die Familie Schneider. Estamos ante algo que podríamos denominar un "doppelgänger arquitectónico". En la obra Die Familie Schneider duplica dos casas típicas londinenses, en las que aparecen dos parejas gemelas interpretando los quehaceres cotidianos, y el visitante es invitado a entrar individualmente en cada casa (figuras 5 y 6). Como comenta Schneider, después de ver la segunda casa con idénticos espacios e idénticos habitantes: "El espectador necesariamente se observa a sí mismo, está al lado de sí mismo. Camina por la casa cerca de él mismo" (Schneider et al., 2006, p. 155).

Andrew O'Hagan señala que hay algo criminal en esas casas. Desde la pintura brillante hasta la disposición de los muebles y los elementos de comodidad de las casas se convierten en algo desconcertante, sobre todo el hecho de tener la chimenea apagada. Y de esta manera comenta: "Uno siente que había algo en la atmósfera que era al mismo tiempo terriblemente familiar y profundamente grotesco" (Schneider et al., 2006, p. 159). 


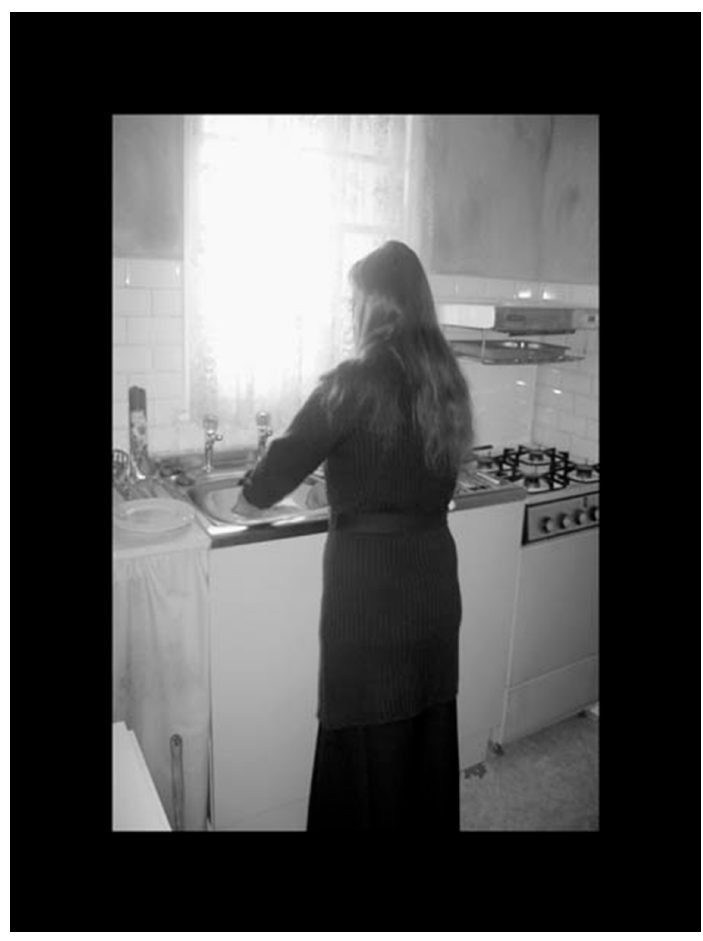

Figura 5. Gregor Schneider, Die Familie Schneider. Actuación en vivo, Walden Street No. 16, Londres, Gran Bretaña, 2004. Fuente: https://tinyurl.com/4xabw2fx 


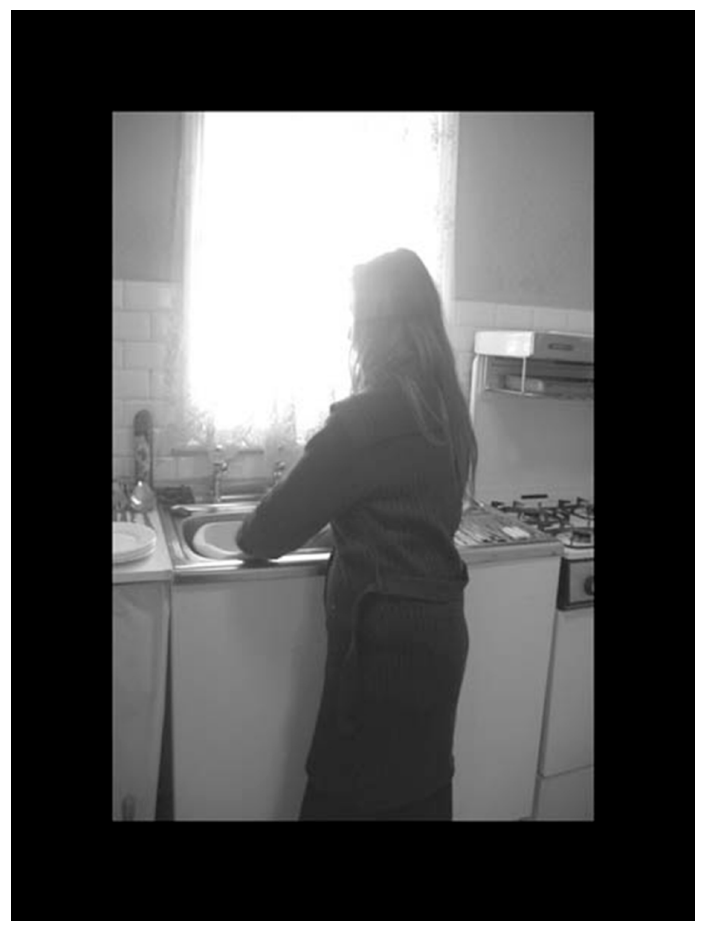

Figura 6. Gregor Schneider, Die Familie Schneider. Actuación en vivo, Walden Street No. 14, Londres, Gran Bretaña, 2004. (C) Gregor Schneider/VG Bild-Kunst. Fuente: https://tinyurl.com/4xabw2fx

El duplicado no aparece como una réplica, sino como una inversión espacial, como un negativo perceptivo que se enfrenta al paso del tiempo y a la desaparición del entorno. Con el "dopplegänger arquitectónico" Schneider contrasta al espectador con sus propias memorias reprimidas, y crea unos vacíos que producen ansiedad y extrañamiento al espectador. De esta manera: 
La praxis de Gregor Schneider es diferente de la del ready-made: con sus propias manos reproduce habitaciones existentes en el mismo lugar y, posteriormente, para fines de exposición, reconstruye estos en otro lugar. De este modo, se hace cargo y afirma un edificio existente, buscando constantemente una conexión con lo que no es, con un nivel extraño más profundo que cuestiona fundamentalmente la posibilidad existencial de la vivienda, de encontrar refugio en una casa. (Loock, 2002, p. 139)

\section{El doble espacial: trauma y memoria}

La representación en la obra de Schneider reactiva el concepto de "memoria". En Haus $u r$, Schneider habilita la remembranza de un espacio anterior que está oculto, pero que reverbera en el ambiente. Para representar el espacio anterior Schneider lo disfraza bajo la apariencia de un nuevo espacio, pero en realidad se trata de una reconstrucción, de un nivel, representado de manera escalonada en el mismo lugar.

Podemos entender la acción de Schneider en su contexto. La rehabilitación constante de la casa se produce en un lugar que sufrió la devastación de los bombardeos de la Segunda Guerra Mundial. En la zona de Rheydt, el 80 por ciento de las casas fueron arrasadas. Schneider recurre constantemente a la memoria de la casa familiar como lugar de acción. En su contexto, Haus u $r$ nos remite a la memoria del lugar o como señala Mark Crinson (2005) a la "memoria urbana". De esta manera, Crinson atribuye un antropomorfismo al paisaje de la ciudad capaz de recuperar la memoria del lugar a través de la reconstrucción:

La memoria urbana puede ser un antropomorfismo (la ciudad que tiene una memoria), pero más comúnmente se puede connotar la ciudad como un paisaje físico y una colección de objetos cuyas prácticas permiten aflorar los recuerdos del pasado. Estos recuerdos están encarnados en los restos de la construcción y reconstrucción secuencial de la ciudad. (2005, p. XII)

Y, eso es exactamente lo que hace Schneider en su obra, centrar su trabajo en la percepción antropomórfica del espectador, en una incesante reconstrucción 
de un espacio habitacional. Su trabajo también habla de una arquitectura simbólica de la ciudad en el contexto histórico alemán de posguerra, donde las huellas y los fragmentos de la destrucción dieron paso a la confrontación con la memoria colectiva. La memoria colectiva, según Halbwachs (2011), opera como contraste a la historia, que es una racionalización del pasado. Esta memoria reúne grupos humanos y los relaciona con su identidad, con "los espacios físicos y los sucesos anteriores", y muchas veces como elementos fundadores de las identidades. La memoria colectiva es entonces, para Halbwachs, sensible a la colectividad espacial de la ciudad.

Estas teorías fueron recogidas por Aldo Rossi (1982) en su libro La arquitectura de la ciudad, que refutó las teorías modernas del urbanismo y la reconstrucción de las ciudades después de la Segunda Guerra Mundial. Rossi propone la memoria de la ciudad a través de sus edificios, en la que la ciudad es recordada a través de los edificios y que estos son semejantes a la sensibilidad y la experiencia de las memorias de la mente humana.

En esta dialéctica entre memoria e historia se encuentra el trabajo de Schneider. Esta se reproduce en "permanente evolución y sujeta a la dialéctica entre el recuerdo y el olvido" (Crinson, 2005, p. XIV). En cierta medida, el trabajo de reconstrucción de los espacios de Haus u $r$ son una crítica simbólica al movimiento de reconstrucción de los espacios urbanos y sus edificios en la posguerra, que Christine Boyer (1996) define como "la representación pictórica del espacio y el tiempo" (p. 3).

Contra esta representación idílica de los espacios fragmentados de la historia, y como respuesta a la representación del capitalismo triunfante de posguerra que ha reprimido lo subjetivo y ha instrumentalizado el pasado, podemos encontrar la misma idea de reconstrucción, duplicación psicológica y espacial de Schneider. Encontramos la idea misma de la reconstrucción en el acto de 
conversión de un espacio privado en un espacio participado y público, donde no se contraponen "los estilos ficcionales y los comportamientos imaginarios" (Boyer, 1996, p. 4) que ha producido la institución arquitectónica y urbanística de la reconstrucción de posguerra.

El trabajo de Schneider fractura la narrativa temporal y propone Haus u $r$ como el inconsciente simbólico del trauma de la ciudad, donde articula una temporalidad espacial fantasmal, plagada de vacíos y discontinuidades. Como apunta Daniela Zyman, Schneider se ha esforzado continuamente para trabajar en una desaceleración de la experiencia, la percepción y la temporalización del espacio. En este sentido, sus espacios "Haus u r o Die Familie Schneider son tentativas, entre otros, de hacer evidente la temporalización del espacio, para reproducir artificialmente las 'huellas del tiempo' (por medio del arte), ergo, creando una conciencia espacial del propio concepto de ruinas" (Schneider et al., 2007, p. 15).

A este respecto, conviene mencionar las observaciones de Dylan Trigg (2009) sobre el tiempo, el espacio y la memoria, en la medida que concluye "que la aparición de la ruina, entendida desde el punto de vista fenomenológico, permite acercarnos al espacio-tiempo de un trauma en términos de una lógica de fantasmas y vacíos" (p. 87). En efecto, las estrategias de representación que incorpora Schneider transitan muy sutilmente entre ilusión y ficción, entre realidad e imaginario, así como entre lo factual y los discursos construidos. De este modo, convoca dialécticamente lo que Pierre Nora (1984) Ilamó "el lugar de la memoria". Dotando a sus espacios de una metafísica del lugar que incorpora una atmósfera siniestra y fantasmal, donde la reverberación y las resonancias mórficas se adueñan del espacio.

Esta táctica de alienación espacio-temporal incorpora en su discurso el trauma como elemento forcluído, interpretando esta forclusión en el sentido que habla 
Trigg (2009), en el "que los sitios del trauma articulan la memoria precisamente a través de la negación de una narrativa temporal continua" (p. 87). Así en su trabajo, Schneider, como habíamos planteado antes, parte de la reconstrucción de las ruinas y de la reconstrucción de la experiencia traumática, tanto de la Alemania de posguerra como de la memoria familiar en el caso de Haus $u r$.

Para ello, Schneider articula metafóricamente la reconstrucción de los fragmentos de las ruinas de la casa, a través de la duplicación de los espacios. La temporalidad de los espacios al duplicarse y reconstruirse se ralentiza, es decir, se encuentran suspendidos en su narrativa temporal, porque la reconstrucción en la obra de Schneider evoca un pasado espacial y temporal que retorna en forma de lo ya visto. $Y$ retorna como algo reprimido en el sentido freudiano, que ahora emerge de nuevo desde el pasado.

El espectador no puede seguir la lógica temporal y espacial por el atrapamiento interior al que está sujeto, y por los efectos desorientadores que le produce la confusión entre espacios exteriores e interiores; es decir, a partir de la idea de "vacío" que provoca al espectador la ilusión de percibir la copia, el doble como el espacio original. Y es la propia performatividad del espectador la que activa un anacronismo perpetuo, ya que los espacios duplicados quedan atrás en el espacio y en el tiempo, y son idénticos a los reconstruidos. Es decir, la percepción que tiene el espectador sobre los espacios se basa en la especulación sobre algo desconocido. Parece como si el espectador, ante la obra de Schneider, sitúa su percepción en un estado de constante aplazamiento, que suspende de alguna manera el presente.

La repetición de las habitaciones se asemeja a un movimiento hacia dentro de la casa, como una forma de acercarse a esa idea de vacío que comenta Schneider. Emergen así las memorias pretéritas, impregnando el espacio de una temporalidad suspendida, que Schneider provoca con un estancamiento 
temporal en la casa de su juventud. Aunque cambien los espacios, estos se desarrollan en la misma casa y siempre negocian con el pasado, con la memoria de la casa. El espectador se encuentra ante esta dialéctica de espacio presente y espacio pasado, entre la construcción y la destrucción, entre la fragmentación de la ruina y la recomposición del lugar que genera una reconfiguración entre presente-pasado y una especulación entre espacio, trauma y memoria.

Siguiendo a Trigg, los lugares del trauma son, ante todo, lugares de la memoria y sin los lugares no habría la posibilidad de recordar. Trigg desarrolla la relación entre memoria y lugar para entender la significación de los lugares y el cambio que sufre un espacio cuando se convierte en lugar, cuando es encarnado por el cuerpo, para así "formar una alianza en la que la memoria es la facultad por sí misma de estar en el lugar" (2010, p. 5).

De esta forma, Trigg señala la espacialidad de la memoria y la interdependencia entre memoria y espacio. Es decir, los lugares representan la memoria, en la medida que llegan a ser un punto de referencia central en la persona, que desarrolla una relación material y temporal con los espacios vividos. La casa es el espacio central del "yo" donde retorna siempre el sujeto, y sin la activación de la memoria en los lugares "la memoria misma ya no tendría un papel que desempeñar en nuestras vidas conscientes" (Trigg, 2012, p. 1).

Para Trigg, la formación de la memoria en el contexto social es otorgada por el poder del lugar, que se manifiesta en forma de "monumentos y sitios de trauma", abrazando la temporalidad del presente y el pasado. A través del trauma, la memoria del lugar rompe la unidad espaciotemporal que el sujeto tiene sobre el espacio vivido. Y se convierte en una percepción que permite las lagunas temporales, los vacíos espaciales y los espectros del lugar. 
El trauma, según Trigg, cuestiona la unidad temporal y espacial de la memoria del lugar. $Y$ en este sentido, la esencia de la memoria del lugar, es decir el hogar, se convierte en algo diferente y definido por lo que es ausente, y lo que es interrumpido temporalmente. La centralidad, el confort y la seguridad del hogar pierden su efecto. Y aquí podemos conectar de alguna manera las ideas planteadas por Trigg con la definición de la casa en Haus u r, como algo desacogedor, uncanny, como una casa desestabilizada y desfamiliarizada. La conexión del trabajo de Schneider con las historias traumáticas de la ocupación Nazi de Alemania se nos presenta como ineludible.

La diferencia entre un lugar de memoria como las ruinas y un lugar traumático es que en el espacio del trauma no hay posibilidad de encontrar huellas físicas reales del trauma, porque se necesita el sujeto superviviente y se crea una laguna entre el espacio y el tiempo del lugar. En el espacio del trauma se produce una experiencia no reclamada, no ligada a la materialidad y a la temporalidad de las ruinas, sino a las voces subjetivas de los que padecieron el trauma (Trigg, 2009, p. 88).

Igual como ocurre en los espacios duplicados de Schneider, lo que está presente en el espectador es la ausencia y la no experiencia del espacio anterior. La tensión que se produce en estos espacios es similar al proceso de "encarnación de la desaparición" en los lugares de la ruina y el trauma, como así propone Trigg. Porque de lo que se trata en ambos casos es intentar reconstruir la experiencia y la memoria del lugar. Si prestamos atención a los postulados de Trigg (2009), vemos que los espacios de Schneider están rodeados por un halo de inquietud y espectralidad, y "lo que se experimenta es menos un fragmento directo de una narrativa rota, y más un murmullo del lugar donde una vez existió esa narrativa" (p. 99). 
Del mismo modo, respecto a las ruinas modernas, Dylan Trigg insiste en que son una atracción y se unen visceralmente con nuestros sentidos, especialmente por las vibraciones que hacen los materiales y su decadencia. Nuestro sentido de estar en el mundo se altera al experimentar estos lugares de decadencia material. Y uno de estos lugares principales, que constituyen esa experiencia como lugar que da sentido a nuestras memorias, es nuestra propia casa.

La memoria de los lugares, entonces, se transforma a través de la idea de la decadencia, la ruina y el trauma. Esta plena facultad de habitar y dar sentido a nuestra propia existencia está relacionada con la casa como lugar de revelación de las memorias. De esta manera, podemos detectar en el trabajo de Schneider esa atracción de las ruinas que él intenta reconstruir una y otra vez, representadas también por las ruinas de la Alemania de posguerra.

Podríamos conjeturar, entonces, que uno de los motivos que impulsan a Schneider a duplicar y reconstruir espacios es la contextualización en la reconstrucción física y real de las ruinas producidas por la Segunda Guerra Mundial. Como él mismo ha declarado en muchas ocasiones, Haus u $r$ es en sí misma una reconstrucción de posguerra. Después de la Segunda Guerra Mundial, la memoria histórica y material de muchas ciudades alemanas desapareció dejando tras de sí ruinas, y una experiencia traumática que Schneider ha reflejado de manera consciente o inconsciente en su trabajo.

Schneider contextualiza su trabajo como sitio de memoria y trauma que articula la memoria del lugar. De hecho, las formulaciones de una "memoria nacional" se encuentran en este "doppelgänger arquitectónico", sutilmente cifrado; en la medida que su trabajo se desarrolla dentro de la crítica espacial y temporal insertada también en la historia como discurso y como remodeladora de espacios y tiempos. 


\section{Conclusión}

El trabajo de Schneider está compuesto, entre otros, de los elementos que hemos debatido en este texto, en el que la memoria, la historia, el extrañamiento y lo forcluído encarnan el significado y la experiencia de su práctica artística. La representación artística que practica Schneider se basa en la elaboración de unos códigos simbólicos ligados a la idea del lugar y el espacio. Su discurso evoluciona con la transformación espacial y con las técnicas de teatralización a modo de instalación escultórica. Para ello, una constante experimentación es la clave que posibilita la reconstrucción de la memoria. Sin embargo, esta se presenta, como hemos indicado, sobre la idea de una memoria nacional, de un "trauma nacional" que condiciona el significado de su discurso.

De este modo, el trauma y la memoria nacional se nos presenta de manera contradictoria ya que, como argumenta Ernst Van Alphen, una de las causas del trauma precisamente es la incapacidad de la experiencia y la memoria de lo que provocó realmente el trauma (Bal et al., 1999, p. 26). Esta "experiencia fallida" que significa el trauma, solo es posible recuperarla o reconstruirla en su discursividad mediante la duplicación y el "doppelgänger espacial", y que incluye en sí mismo la temporalidad del lugar.

Pero, siguiendo las investigaciones de Trigg sobre la memoria, observamos que no solamente el espacio puede desencadenar la memoria, sino que la cinestesia performativa del visitante es esencial para dar cabida a la visualización de la memoria traumática. Y como apunta Trigg (2012), el espacio se convierte en lugar concreto mediante la participación del cuerpo y la sensibilidad corporal de la cinestesia hace posible la memoria del lugar (p. 103). Aquí, es donde interviene la performatividad del espacio en las instalaciones de Schneider. Es a través de la exploración subjetiva e individual, in situ, de los espectadores cuando sus instalaciones espaciales devienen legibles. 
Así, concluimos con los resultados de esta investigación apuntando las aportaciones que hemos destacado en el trabajo de Schneider. Y quizás la que defina de una manera más clara su procedimiento de trabajo sería la incorporación del espectador, activando conjuntamente elementos como la arquitectura, el espacio, la performance y la instalación. El espectador produce la obra de Schneider cuando su trabajo le brinda la posibilidad de experimentarlo de manera individual, y esta es una de las razones por las que la obra de Schneider es conocida como una de las más grotescas y uncanny en la actualidad.

Schneider confronta al espectador con sus propios miedos, sus deseos, y sus memorias a través de la temporalización de los espacios que reconstruye y manipula. La obra enfrenta al espectador con la posibilidad de revertir el espacio-tiempo acelerado de nuestra sociedad capitalista, de cambiar su significado y de someter a escrutinio nuestra experiencia de los gestos y comportamientos cotidianos, de nuestras seguridades y vulnerabilidades, de nuestras oscuridades e iluminaciones, de nuestras dualidades permanentes. Como señala Daniela Zyman, hablando de la obra de Schneider, el silencio y la pausa del espectador son necesarios para construir, percibir y habitar sus espacios (Schneider et al., 2007, p. 15).

\section{Agradecimientos}

Este trabajo está incluido en el proyecto de investigación "El espacio articulado: contextualizaciones en el arte contemporáneo, espacialidades y temporalidades en la producción artística actual". HAR2015-64106-P (MINECO/FEDER). El documento está financiado por el "Programa Estatal de Fomento de la Investigación Científica y Técnica de Excelencia, Proyectos de I+D, del Ministerio de Economía y Competitividad" del Gobierno de España. 


\section{Referencias}

Auslander, P. (2003). Behind the Scenes: Gregor Schneider's Totes Haus u r. PAJ: A Journal of Performance and Art, 25(3), 86-90, https://doi.org/10.1162/152028103322491719.

Bachelard, G. (2012). La poética del espacio. Fondo de Cultura Económica.

Bal, M. (1999). Quoting Caravaggio: Contemporary Art, Preposterous History. University of Chicago Press.

Bal, M., Crewe, J. V. y Spitzer, L. (Eds.). (1999). Acts of Memory: Cultural Recall in the Present. Dartmouth College.

Boyer, M. C. (1996). The City of Collective Memory: Its Historical Imagery and Architectural Entertainments. MIT Press.

Crinson, M. (Ed.). (2005). Urban memory: History and amnesia in the modern city. Routledge.

Dionne, C. (2003). Gregor Schneider: Des mouvances limitrophes. Revue d'art contemporain ETC, 63, 28-34.

Enwezor, O. (Ed.). (2006). Lo desacogedor: Escenas fantasmas en la sociedad global. BIACS.

Freud, S. (1919). The Uncanny (J. Strachey, Trans.). The standard edition of the complete psychological works of Sigmund Freud (Vol. 17), pp. 217-256.

Halbwachs, M. (2011). La memoria colectiva. Miño y Dávila Editores.

Kobayashi, H. e Inoue, A. (2014). Interview with Gregor Schneider. Magcul. http://magcul.net/ focus/gregor_schneider2sw_page/

Lacan, J. (1988). The seminar of Jacques Lacan. Polity Press.

Loock, U. (2002). Gregor Schneider, The Dead House Ur. Parkett, 63, 138-151.

Moriente, D. (2007). La ficción distópica de Gregor Schneider. Anuario del Departamento de Historia y Teoria del Arte, 19, 189-208.

Nora, P. (1984). Les Lieux de mémoire. Gallimard.

Rossi, A. (1982). The architecture of the city. MIT Press.

Schneider, G., Loers, V. y Loock, U. (2011). Punto muerto: Gregor Schneider. CA2M. 
Schneider, G., O’Hagan, A., y Tóibín, C. (2006). Die Familie Schneider. Artangel.

Schneider, G., Siebold, A., Pfeffer, S. y Zyman, D. (2007). Gregor Schneider: 19-20:30 Uhr 31.05.2007 7-8:30 pm 05.31.2007. Verlag der Buchhandlung Walther König.

Trigg, D. (2009). The place of trauma: Memory, hauntings, and the temporality of ruins. Memory Studies, 2(1), 87-101.

Trigg, D. (15 de Enero 2010). Architecture and Nostalgia in the Age of Ruin. University of Bath, Architecture Department.

https://www.academia.edu/208447/Architecture_and_Nostalgia_in_the_Age_of_Ruin

Trigg, D. (2012). The memory of place: A phenomenology of the Uncanny. Ohio University Press.

Williams, G. (2010). Doubling: Gregor Schneider interviewed by Gilda Williams. Art monthly, $340,1-6$.

Cómo citar: Segura Cabañero, J. y Simó Mulet, T. (2021). La memoria del lugar: lo extraño y la forclusión en Gregor Schneider. Revista KEPES, 18(24), 107-136. https://doi.org/10.17151/kepes.2021.18.24.5 